\title{
Service Interaction Flow Analysis Technique for Service Personalization
}

\author{
Olli Korhonen ${ }^{1}$, Anna-Liisa Syrjänen ${ }^{1}$, Marianne Kinnula ${ }^{1}$, Minna Isomursu ${ }^{2}$, and \\ Kari Kuutti ${ }^{1}$ \\ ${ }^{1}$ University of Oulu, Oulu, Finland \\ ${ }^{2}$ IT University of Copenhagen, Copenhagen, Denmark \\ \{olli.korhonen, anna-liisa.syrjanen, marianne.kinnula,kari.kuutti\}@oulu.fi \\ miis@itu.dk
}

\begin{abstract}
Service interaction flows are difficult to capture, analyze, outline, and represent for research and design purposes. We examine how variation of personalized service flows in technology-mediated service interaction can be modeled and analyzed to provide information on how service personalization could support interaction. We have analyzed service interaction cases in a context of technology-mediated car rental service. With the analysis technique we propose, inspired by Interaction Analysis method, we were able to capture and model the situational service interaction. Our contribution regarding technologymediated service interaction design is twofold: First, with the increased understanding on the role of personalization in managing variation in technologymediated service interaction, our study contributes to designing service management information systems and human-computer interfaces that support personalized service interaction flows. Second, we provide a new analysis technique for situated interaction analysis, particularly when the aim is to understand personalization in service interaction flows.
\end{abstract}

Keywords: Service personalization, service interaction, service management information system, interaction flow, interaction analysis

\section{Introduction}

Multilayered information and communication technology (ICT) affords delivering of services by sophisticated interfaces that are able to support complex service interaction. Complex service interaction is difficult to capture, analyze, outline, and represent for information systems research and design purposes. During the last decades the analytical interest has focused on perceived quality [47] and how it can be measured and attached to service providers and customers who, with their differing needs and goals, share service interaction and are seen as co-creating its value $[8,9,11,37]$. Existing research on service management emphasizes that we should pay critical attention to the service quality formed across all moments of contact with organizations providing service $[8,13,36]$. We argue that there is still lack of understanding of how automated or technology supported service management could be implemented on the 
level of a unique service process for achieving a personalized service flow which adapts to the needs of the actors taking part in the service delivery process.

Over decades, the idea of providing standardized services while treating each customer as a unique person has remained in the service marketing literature [44]. Service personalization means adaptation of service clerks' interpersonal behavior and interaction in a way it suits a particular customer's needs [15]. Today that interaction is often enhanced with technology, and the interaction is mediated through interfaces and devices [14]. In the human-computer interaction (HCI) and information systems (IS) fields, personalization interests join in the question of how to provide services online, in different contexts, and serving the needs of different kinds of users; the key interest to researchers being the delivery component, the interface [3] by which different interaction and service problems manifest themselves. [3] argues that there are still shortcomings around solid theoretical perspectives on which parts of service delivery should be computer-driven and which parts should involve humans, and also lack of research on evaluating different ways of how human service interaction could be delivered and which media would be appropriate in mediating it in different interaction situations. Accordingly, in information systems research and design there is an ongoing change from focusing solely on professional and managerial users to more heterogeneous audiences [3] including children [18], elderly, and persons with memory problems [26], together with many other user groups with special ICT needs.

Interaction and collaboration studies have shown that when compared to direct interaction between people in situ, use of technology in the interaction can make some issues even more visible [20, 27, 36]. One such instance is situatedness of action, seen as manifesting variation and thus necessitating understanding of different viewpoints of actors, in a situation where people interact without having a good model of each other's action, skills, resources, or location or other influential factors of that particular situation $[40,43]$. While related studies have focused on how people refer to other people, objects of interest, and environment, and share information in documents or tangible things $[12,20]$, studies related to personalization of service interaction itself are scarce.

In this study, our aim is to understand and analyze variation of service interaction flow for personalization of service interaction, and to propose and demonstrate a new technique for doing that. Therefore, we ask as our research question: How can we recognize and capture variation of personalized service flows in technology-mediated service interaction? To answer this, we analyzed interaction in agreement-based car rental service where the service provider utilizes video-mediated communication (VMC) [12] in their service kiosk interfaces. Interaction Analysis method [19] was applied to investigate the human activities, and to identify and illustrate the variation in service interaction. The kiosks are highly interactive computer-based information systems that are located in public areas [17]. In our service case, these kiosks enable technology-mediated service encounters between service clerk and customer [14] and service provider's service management information system (SMIS) supports personalization of service interaction to meet the needs of both the service provider and each individual customer. Next, we discuss related research on personalization in service context, how it has been considered in service systems design, and how variation of service flows has been approached for service interaction analysis. After that, we describe our methodology, summarize and discuss with our results. We 
conclude by discussing the implications of our results to IS and HCI, limitations, and paths for future work.

\section{Related Research}

\subsection{Personalization of Services}

Service personalization is defined as any behavior occurring in a service interaction intended to individuate the customer [44]. In service literature, personalization is defined as adaptation of service clerk's interpersonal behavior and interaction in a way it suits a particular customer's preferences $[10,15]$. In technology mediated services, this behavior and interaction is supported by an information system. Service personalization is often intertwined with another concept used to tune a service to individual customer preferences: service customization that focuses on configuration of the service content [10]. Both personalization and customization can be done in the interaction between service clerk and customer $[15,48]$.

Personalization has been conceptualized as option personalization, programmed personalization, and customized personalization. The first one focuses on the service outcome and latter two on service process [44]. Option personalization [44] means customization of service outcome, where customer configures the service, for example, by choosing a set of components from a pre-set menu to create a unique service [10, 48]. This can vary from use of fully customized unique service packages to services that include both customized and standardized components [21]. Service component here means a resource used in service delivery - be it human resource, ICT resource, or information. Use of SMIS allows real-time construction and support of a service flow to provide unique service packages with best available resources, as is done for digital services with web service composition [28].

Programmed personalization and customized personalization focus on personalizing the interactive process of the service and the way service is delivered for individual customer [10,44]. Example of programmed personalization is the use of personalized small talk, where customer is called by name during the interaction. Customized personalization on the other hand focuses on interpersonal behavior adaptation, such as using similar dialects or vocabulary to personalize the service process [44].

In service systems design, personalization can be integrated in different virtual systems (e.g. online systems/websites), physical systems (e.g. human intervention, delivery, logistics) and integration systems, which should enable the seamless function of other systems [39]. As most of the systems utilize human-computer interaction, quality of services and potential for personalized service interaction is in effect dependent on it. In interactive services, service quality has been addressed to service providers' customer orientation, customers' service expectations for and experiences in services, and to the interaction by which quality service involves people, technologies, and organization and attaches economical costs and practical benefits to both sides [8]. Therefore, the sensitive point in service systems personalization is the customer-service provider relationship, depending on such factors as how well the 
needs of individuals and the suitable interaction styles for each of them are understood in service delivery.

In this paper, we propose that personalization is a technique for managing variation of service interaction flows when there is a need to adapt service interaction to the needs of actors participating in service delivery, such as the customer, front-stage clerk, and the organizations delivering the service. Advanced IS can support personalization better if the service interaction alternatives and logic are analyzed and understood by the designers.

\subsection{Analyzing Service Interaction Flow}

Traditionally, different aspects of variation in service interaction flows have been analysed in studies, which have aimed to challenge existing ways of thinking, analysing, and designing interactive technological systems [24, 27, 40, 43]. Regarding service-oriented thinking, [47] argue that standardization of the output of a service is much more difficult compared to standardization of goods. Contemporary services are more like "activities" or "processes", and rather than goods customers buy "offerings". In this line of thought, variation embodies harmony with "the individualized, dynamic demand of the customer" [47]. The authors argue that companies should construct also their goods to be more service-like by customizing the output according to the varying standards of consumers, and they show that companies that have realized this have gained competitive advantage [47].

Varying demands for use are well recognized in design activities where the need for supporting personalized service flows is seen as an existing and enduring challenge in everyday decision-making. Along with changing activities and conditions [5, 7, 31], variation usually entails continuous work with their reorganization, as well as distribution of collective capabilities [33]. Accordingly, analysis and design involve the capacity of capturing, analysing, outlining, and representing the essential situated aspects [42]. Design methods are situated by nature and can be supportive regarding different instances of situatedness, including situated knowledge, action and learning, and situating context [38].

According to [41] the basic idea in situated analysis is to challenge actors' assumptions on how activities are carried out by making visible something that is not apparent but essentially influences their working. Many invisible forms of work were recognized with a conclusion of "the better the work is done, the less visible it is to those who benefit from it" [41]. Hence, representations such as service interaction visualizations by texts, snapshots, state transition patterns, graphs of time-line, statistical mode, and state transition probability map [29], and results from ethnographic analysis and interaction analysis on using VMC in multidisciplinary medical team meetings [20] emphasise both the importance and the challenges of visualizing variation in multi-actor interaction.

[32] argue that service design is a growing practice and there is a continuous need for new tools, for making sense of the intangible and tangible qualities of services. [35] in turn maintain that service design is an emerging field where methods are not yet well established. Service process flows can be modelled with several methods and approaches. Basically all modelling techniques applicable to processes in general can be used to model service process flows in particular, such as IDEF diagrams or Petrinets 
[6], structured workflow modeling [22], and business process modeling in general [1]. More recently, service design methods addressing not only the service provider viewpoint, but also the viewpoint of customer and network of actors needed in the service process have emerged [46]. For example, customer journey method [50] can be used to describe the touchpoints between a company and its customers when experiencing a service. Service blueprinting [4] takes a customer perspective for visualizing the service processes. The technique can be also applied in a service situation with multiple actors with different motivations [49] and for designing adaptive services [25]. However, as service blueprinting visualizes all actions that are needed for a service to function [49] the support for capturing and modeling variation with that technology requires multiple blueprints to cover all the potential variations in the service interaction. Therefore, we propose an analysis technique to capture and model variation in service interaction.

\section{Methodology}

\subsection{Context}

Our study was conducted in 2012 with a service provider that offers agreement-based car rental services and had recently extended to a new kiosk-based service, configured as a special HCI setup for online face-to-face (F2F) service. The kiosk uses a secure data transmission connection over the internet between the provider's service management system and service clerks' and customers' locations. Interaction between a service clerk and a kiosk customer is mediated by VMC-based interfaces together with remote-controlled kiosk system's devices and peripherals especially designed for this type of service. These peripherals include functions for secure authentication, service agreement, payment, and releasing of car keys. The delivery model resembles video banking [34] and supports variation in communication by VMC.

\subsection{Data}

Our data consists of 16 video-recorded service processes of individuals (id1-id16; ages 22-63; seven women, nine men; Finnish and English languages, non-native) who used the service kiosk in a public space while the front-stage clerk worked over the internet following the service provider's standard rental procedure. All participants were new car renters for the provider and the analyzed interaction processes went through the standard phases of car renting process included in the service description. Eight phases for a typical car rental service process have been identified in [45]. However, as some of those phases consist of multiple acts (e.g. phase 6 includes personal details, signing the rental agreement, and paying), we decided to distinct the acts into separate phases of their own, resulting in 11 interaction phases in overall. The phases are: starting, identifying needs of the customer, discussing requirements, charging, discussing possible add-ons, identification of the customer, authentication, payment, making of a rental agreement, delivery of car keys, and ending the service process (Table 1). 
Table 1. Interaction phases and interface components.

\begin{tabular}{ll}
\hline Interaction Phase & Interface Components Used \\
\hline Start (greetings) & \\
Needs & VMC by cameras \\
Requirements & Microphones \\
Charge & Video displays \\
Add-ons & \\
\hline Identity & \\
Authentication & VMC by cameras \\
\hline \multirow{2}{*}{ Payment } & Document camera \\
\hline \multirow{2}{*}{ Agreement } & VMC by cameras \\
& Credit card reader \\
\hline \multirow{2}{*}{ Delivery } & VMC by cameras \\
\hline Ending (parting) & Scanner \\
\hline
\end{tabular}

The rental process lasted for 8-12 minutes and users were interviewed before (general background information) and after the rental process (evaluation of the experience related to service process). None of the participants had used this type of kiosk service interface before but all of them had used face-to-face services and selfservices. Nine of them were first-time car renters and seven had prior experience of car rental process. We used parallel columnar transcript method [19] for transcribing verbal and nonverbal behavior of the use situations, making it possible to capture kiosk users' interaction at the kiosk interface, and the service clerk's interaction via VMC devices.

\subsection{Analysis Procedure}

Our analysis was inspired by work on situated interaction between the participants of a particular social situation [19]. However, instead of identifying regularities in participants' use of resources [19] in service delivery, our main goal in this paper is to identify and illustrate variation in service interaction. Therefore, our unit of analysis is a single phrase or an act. For instance:

- Service clerk (Sc) says: "Welcome to Xxxxx car rental. How may I help you?" <= this is a phrase. 
- When a service clerk asks from the customer (C) personal details: 'participant showed personal details by setting her business card on a document camera area' $<=$ this is an act.

Based on the order of the phrases/acts and discussed issues, the phrases were compared with the service provider's standard procedure and divided into related phases and shown as flow points $(\bullet)$ in figures below. For instance (excerpt 1$)$ :

1. Sc: "Welcome to Xxxxx car rental. How may I help you?" $<=$ this is related to the start phase $(\bullet)$.

When needed, phrases were further combined so that related answers/complementary questions were counted as a one-content unit. For instance (excerpt 2, excerpt 3):

2. C: "I want ... I want to rent a car." Sc: "OK." $<=$ this is one unit, related to the needs phase $(\bullet)$.

3. C: "I have planned to visit ... to go to (Place), it is a family visit." Sc: "OK, to go to (Place), and would you need a car immediately today?" C: "No, I just need it on the weekend, next weekend." Sc: "OK... the forthcoming weekend, so that would be ...you need it on Friday?" C: "No, on Saturday. ..." <= this is one unit, related to the requirements phase $(\bullet)$.

Correspondingly, multi-content phrases were divided into separate units based on the phases. For instance (excerpt 4):

4. Sc: "It will be five euros additional | but we will charge your credit card?" $<=$ here we have two units, the former is related to the charge phase $(\bullet)$, and the latter to the payment phase $(\bullet)$.

When the answer could not be interpreted to refer to a foresaid content item (e.g. credit card in this case), it was integrated with the latter one. For instance (excerpt 5):

5. Sc: "It will be five euros additional | but we will charge your credit card?" $\mathrm{C}$ answering: "Oh, very fine. Thank you so much!" <= The customer's answer is related to the payment phase $(\bullet)$.

After this outlining, individual rental processes were represented as graphs using the flow points $(\bullet)$, which show how interaction flows between different service phases (e.g., requirements were discussed in several occasions), the lines connecting the flow points showing advancement in time. Finally, the processes were compared by layering flow graphs of four corresponding customer service situations on top of each other (four is the maximum number of cases for the figures still to be readable in this paper). See Figures 3 and 4 as examples.

We consider this degree of precision in representation as appropriate in illustrating relevant aspects of variation in a manner we see useful for systems study and design in practice.

\section{Analysis Results}

Using the standard car rental service process as a frame for describing individual service interactions, we were able to identify and capture variation caused by personalization in service interaction. Service interaction was personalized by actors participating in service delivery, such as verbal variation in flow of service interaction 
as well as sequential variation, for instance, the temporal re-orderings within service phases.

In our analysis, we first represent an example by phrase units, which is one of the common ways of visualizing variation in spoken interaction [19]. Second, by using the flow points defined earlier for the graphs, we exemplify variation in temporal interaction changes by following the content shifts in the service process.

\subsection{Verbal Variation in the Flow of Service Interaction}

Verbal variation in service interaction was recognised as different vocabulary was used in the service process. That is exemplified in the excerpt 3 where the requirements for the starting time of rental were specified by using five different time measurements: "on the weekend", "next weekend", "forthcoming weekend", "Friday", and "Saturday". However, neither the customer nor the service clerk serving her used the exact date recorded into the provider's SMIS or the terms used in the rental agreement. We consider this as epitomizing customer-oriented communication [8] regarding the interaction needs and modes of individual customers; in other words, tuning the communication and interaction style to the specific needs of the customer [44].

The examples manifest different kinds of variation, such as different vocabulary and conventions, used in personalized interaction, showing conceptual variation [40] in interaction modes, and situational variation in action [43]. Despite the variation, the standard service process was loosely followed.

\subsection{Sequential Variation in Flow of Service Interaction}

Sequential variation in individual service process realizations is demonstrated in service interaction flows (Figures 1 and 2) of two participants (id16 and id8).

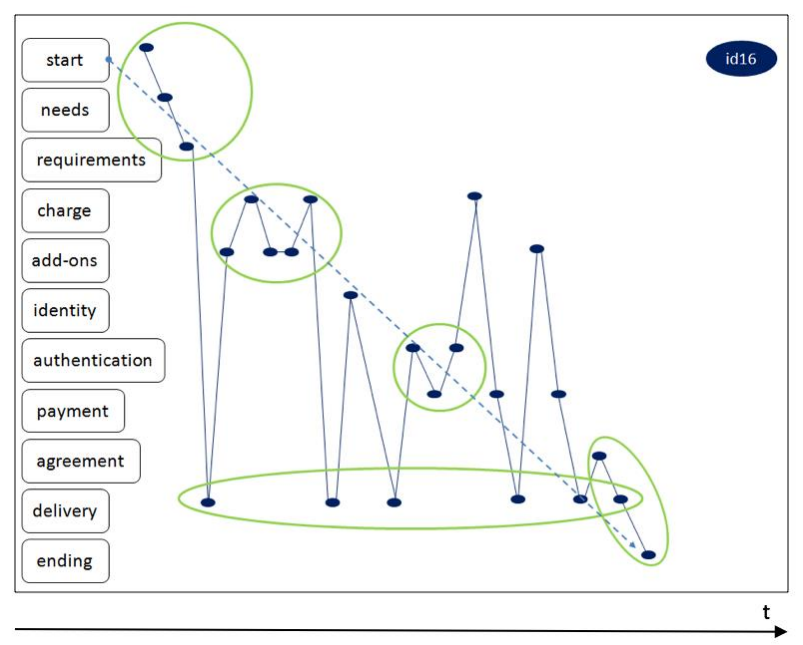

Fig. 1. Service interaction flow of id16 (dark blue steps). 
The linear standard service interaction flow (the blue dotted line in Figures 1 and 2) is most closely followed during the few first steps and at the end of the interaction. After the first steps, the anomaly increases by discussion deviations going to delivery issues, from these to add-ons and charge negotiations, and then back to delivery again, and so on. Steps between add-ons, charge, authentication, and payment show supportive connections and temporal re-organization of the phases in course of the interaction. In Figure 1, shaping of the service outcome for delivery has been an important topic throughout the interaction (six separate steps). In addition, the flow captures variation in total of 24 steps and shows the interaction as progressive: all needed phases were completed and the service could be delivered.

The same is also visible in the flow of id8, though the interaction itself was differently organized (Figure 2). The interaction flow of id 8 has a linear flow of five steps from start to add-ons. After that it proceeded variably going from discussing of charge/add-ons to identity/requirements and authentication/charge. The outcome of the service has been nearly finished in the middle, except the additional service spurt at the end.

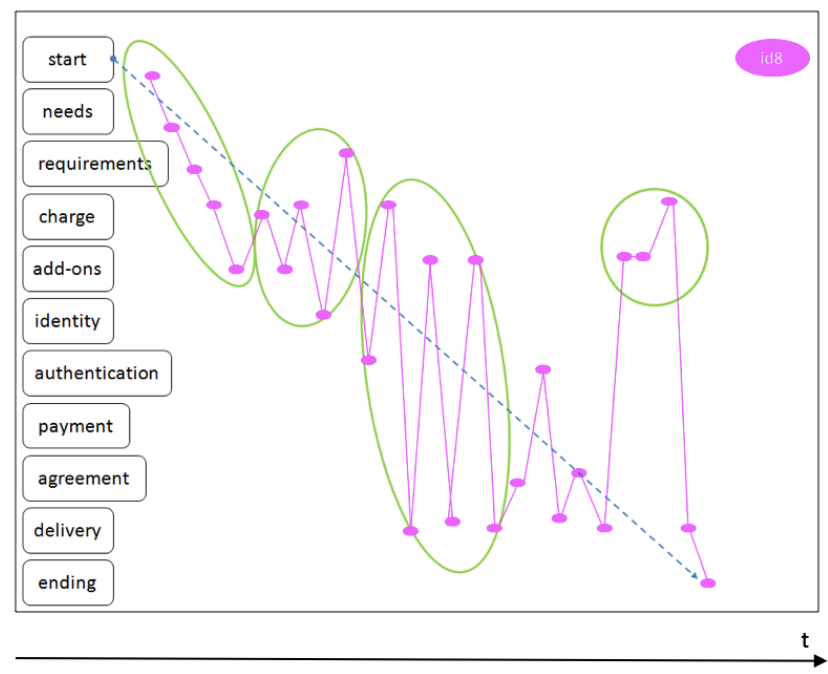

Fig. 2. Service interaction flow of id8 (pink steps).

Individual interaction flows can also be compared with the flows of other customers by layering graphs on top of each other. The flow comparisons enable capturing of the relationship between the varying interaction changes [40] on the one hand, and, on the other hand, the factors affecting each other by the sifting focus of interests of actors [30] in their interaction situation. For instance, from the flow comparison of four individual users (group A in Figure 3) we can see that requirements/charge/add-ons steps have close-knit internal connections but identity, payment, and delivery discussions disperse unevenly. 


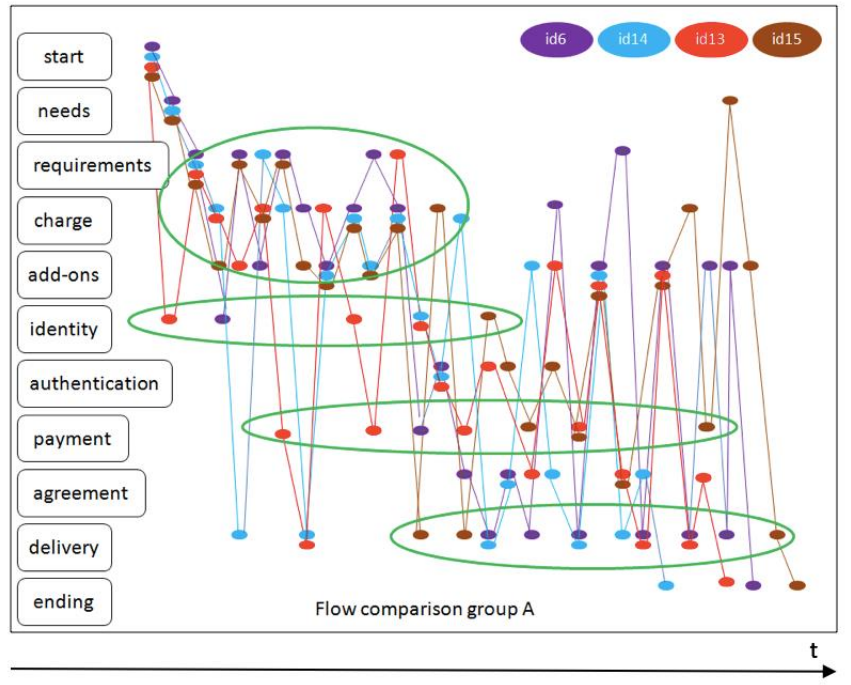

Fig. 3. Comparisons of service interaction flows by a group of four individual users (group A).

From the flow comparison of group B (Figure 4), we can identify the linear starting steps, the repeated, close temporary add-ons/charge connection, and see that throughout the interaction add-ons have been an important factor in shaping of the total service outcome for delivery. These are just few examples of what can be analyzed by the flows.

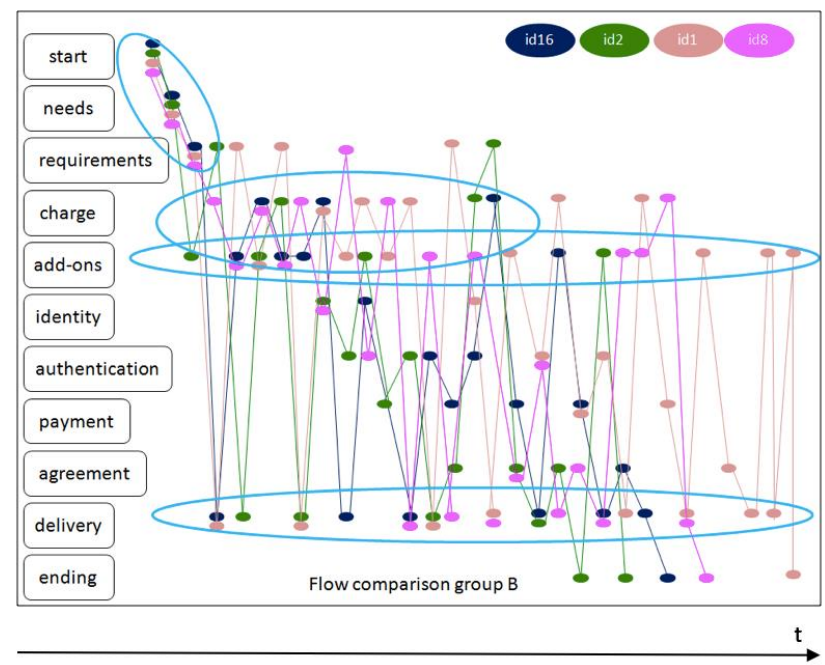

Fig. 4. Comparisons of service interaction flows by a group of four individual users (group B). 
We consider that the comparisons have potential for making generalizations and new observations related to service interactions, for instance when asking what kinds of strategies to adopt for service interaction or process design and how these match with the customers' interaction modes.

Furthermore, when creating understanding on a certain interaction process, we can look at what changes and what does not change in the process. For example, there is only one flow (Figure 3, group A, id13, red) which did not follow the common startneeds-requirements line in the beginning, and one flow (Figure 4, group B, id2, green) with two ending steps. Therefore, we can ask whether variation is more characteristic to certain types of interaction processes, certain service phases, or interaction modes, and what makes the other processes, phases, or modes to be more integrated?

Comparisons also show that in spite of this dynamicity and variation in service interaction, all service situations were performed successfully and produced a personalized service interaction flow and service outcome for each participant. In fact, we were not able to identify any interaction flow that would have followed the standard service process per se or even similarly-ordered interaction flows. However, most participants' interaction flows followed a common line from start to needs to requirements and all the service interactions ended with parting rituals.

As a result, we argue that with the analysis technique presented in this section we have been able to capture and show variation derived from the original situational service activities. In other words, our measures are not separated from the sources; rather they combine essential interaction contents into new forms as situations emerged [23]. We also claim that with this technique we were able to capture personalization of service interaction in the car rental context, and to find shaping points for service outcomes according to the varying requirements of customers, as is suggested by [47].

\section{Concluding Discussion}

This paper inquired how can we recognize and capture variation of personalized service flows in technology-mediated service interaction. This was studied in the context of agreement-based car rental service that uses a special HCI setup for service delivery.

\subsection{Summary of the Results}

Our aim for this study was to understand what implications variation of service interaction flows has for personalization of service interaction. By analysing and modelling interaction between customers and a service clerk, we focused on capturing evidence on how variations of service flows can be made visible in technologymediated service environment. We present an improved analysis technique that models the service interaction flow step by step, adding a visual dimension to the interaction analysis [19] and going in the micro-level in interaction compared to customer journey analysis [50]. With this technique we were able to capture and model variation in personalized service flows, derived from the original situational service activity. Variation can be seen as incorporated on certain levels of situated service interaction, phrases, service phases, and interaction flows. These have been outlined as 1) verbal 
variation in flow of service interaction, and 2) sequential variation in flow of service interaction.

We argue that this technique has the capacity of showing essential elements of service interaction personalization and revealing variation in the personalized service flows, and propose that understanding gained from that can be used for designing service management systems and interfaces that support construction and delivery of personalized service interaction flows. As we have shown with our data, personalization of service interaction flows contributed to shaping of the service process and outcomes, according to the actors' varying needs. In our context, service interaction personalization can be seen as framed by the provider's standard service procedure which, however, became re-constructed by the service clerk who applied it in the actual service interaction, adapting to each unique service situation. Service interaction personalization took therefore place 1) within individual service processes, 2 ) in individual service phases, 3) in temporal reorganization of phases by close-knit internal content connections, and 4) in unique content combinations via several phases.

\subsection{Implications for Research and Practice}

Our findings show that even in a relatively simple service, as illustrated in our case study, a lot of variety between individual service interaction flows took place during service encounters. We claim that this has two implications for practice and research. First, when an information system is used as a resource in a service process, it needs to adapt to the varying nature of actual service interaction flows. In our data, SMIS used by the service clerk allowed entering data in the natural order of the service interaction flow, and it provided consistency checks necessary to make sure that the process was completed successfully. Second, if some parts of the service interaction flow were automated or supported by a service management system, service interaction flow models could be used for implementing automated and real-time service composition and consistency check features similar to methods developed for web service composition [28] aiming at service orchestration done through coordination models [2]. SMIS could compose a service in real-time, adapting to the needs of the actors and context where the service is delivered, thus managing a variety of different contextual parameters [16] relevant from the service personalization point of view.

Our analysis technique can also help researchers and designers to understand interaction flows of services that have not been formalized into a defined model, but have been formulated through experience over time. It can also help both researchers and practitioners to identify different phases in service interaction flows as well as variety in the unique interaction flows of individual customers. Another possibility would be to design which parts of the service are fully standardized and which parts allow more customization [21].

\subsection{Limitations and Future Research}

The results have some limitations to be noted. We studied service interaction in a relatively simple service setup of car rental service. The analysed service interaction flow was completed during a relatively short time, and the service was entirely delivered in interaction between one service clerk and a customer. It may be that new 
insights could be revealed from a more complicated service setup [14], where several service providers work together to compose a holistic personalized service experience. However, we argue that the analysis technique utilized in our study is very versatile and has potential also in the contexts of more complex service research and design areas. For instance, in usability, user experience, and interaction design studies, and when complementing situated design methods for collaborative processes [38]. It would be interesting to see future studies utilizing the technique for deeper understanding of what happens in a service delivery situation where the service delivery spans over a longer period of time and requires collaboration of several actors and service providers.

\section{References}

1. Aguilar-Saven, R.S.: Business process modelling: Review and framework. Int. J. Prod. Econ. 90(2) 129--149 (2004)

2. Barbosa, M.A. and Barbosa, L.S.: A perspective on service orchestration. Sci. Comput. Program. 74(9) 671--687 (2009)

3. Benbasat, I.: HCI research: Future challenges and directions. AIS Transact. Human Comp. Interact. 2(2) 16--21 (2010)

4. Bitner, M.J., Ostrom, A.L., Morgan, F.N.: Service blueprinting: a practical technique for service innovation. Calif. Manag. Rev. 50(3) 66--94 (2008)

5. Bødker, S., Christiansen, E.: Designing for ephemerality and prototypicality. In Proceedings of the 5th conference on Designing interactive systems: processes, practices, methods, and techniques, pp. 255--260 (2004)

6. Bosilj-Vuksic, V., Giaglis, G.M., Hlupic, V.: IDEF diagrams and petri nets for business process modeling: Suitability, efficacy, and complementary use. In Enterp. Inform. Syst II. pp. 143--148 Springer (2001)

7. Büscher, M., Gill, S., Mogensen, P., Shapiro, D.: Landscapes of practice: Bricolage as a method for situated design. Comp. Support. Coop. Work. 10(1) 1--28 (2001)

8. Campbell, C.S., Maglio, P.P., Davis, M.M.: From self-service to super-service: a resource mapping framework for co-creating value by shifting the boundary between provider and customer. Inf. Syst. E-bus. Manag. 9(2) 173--191 (2011)

9. Concilio, G., Deserti, A., Rizzo, F.: Exploring the interplay between urban governance and smart services codesign. Interact. Des. Archit. 20, 33--47 (2014)

10. De Blok, C., Meijboom, B., Luijkx, K., Schols, J.: The human dimension of modular care provision: opportunities for personalization and customization. Int. J. Prod. Econ. 142(1) 16$-26(2013)$

11. Ekelin, A., Eriksén, S.: Citizen-Driven Design: Leveraging Participatory Design of EGovernment 2.0 Through Local and Global Collaborations. In Case Studies in e-Government 2.0. pp. 67--85 Springer (2015)

12. Finn, K.E., Sellen, A.J., Wilbur, S.B.: Video-mediated communication. Lawrence Erlbaum Associates, Hillsdale NJ (1997)

13. Gabbott, M., Tsarenko, Y., Mok, W.H.: Emotional intelligence as a moderator of coping strategies and service outcomes in circumstances of service failure. J. Serv. Res. 14(2) 234-$248(2011)$

14. Glushko, R.J.: Seven contexts for service system design. In Handbook of service science. pp. 219--249 Springer (2010)

15. Gwinner, K.P., Bitner, M.J., Brown, S.W., Kumar, A.: Service customization through employee adaptiveness. J. Serv. Res. 8(2) 131--148 (2005) 
16. Ho, S.Y., Bodoff, D., Tam, K.Y.: Timing of adaptive web personalization and its effects on online consumer behavior. Inform. Syst. Res. 22(3) 660--679 (2011)

17. Holfelder, W., Hebmann, D.A.: Networked Multimedia Retrieval Management System for Distributed Kiosk Applications. In ICMCS pp. 343--351 (1994)

18. Iivari, N., Kinnula, M., Kuure, L.: With best intentions-a Foucauldian examination on children's genuine participation in ICT design. Inform. Technol. Peopl. 28(2) 246--280 (2015)

19. Jordan, B., Henderson, A.: Interaction analysis: Foundations and practice. J. Learn. Sci. 4(1) 39--103 (1995)

20. Kane, B., Luz, S.: Multidisciplinary medical team meetings: An analysis of collaborative working with special attention to timing and teleconferencing. Comput. Support. Coop. Work. 15(5-6) 501--535 (2006)

21. Kellogg, D.L., Nie, W.: A framework for strategic service management. J. Oper. Manag. 13(4) 323--337 (1995)

22. Kiepuszewski, B., Ter Hofstede, Arthur Harry Maria., Bussler, C.J.: On structured workflow modelling. In International Conference on Advanced Information Systems Engineering, pp. 431--445 (2000)

23. Klein, H.K., Myers, M.D.: A set of principles for conducting and evaluating interpretive field studies in information systems. MIS Quart. 67--93 (1999)

24. Kuutti, K., Bannon, L.J.; The turn to practice in HCI: Towards a research agenda. In Proceedings of the 32nd annual ACM conference on Human factors in computing systems, pp. 3543--3552 (2014)

25. Lee, M.K., Forlizzi, J.: Designing adaptive robotic services. In: Proceedings of IASDR'09 (2009)

26. Leinonen, E., Syrjänen, A., Isomursu, M.: Designing assistive and cooperative HCI for older adults' movement. In: Proceedings of the 8th Nordic Conference on Human-Computer Interaction: Fun, Fast, Foundational, pp. 877--882 (2014)

27. Löwgren, J., Reimer, B.: The computer is a medium, not a tool: Collaborative media challenging interaction design. Challenges, 4(1) 86--102 (2013)

28. Milanovic, N., Malek, M.: Current solutions for web service composition. IEEE Internet Comput. 8(6) 51--59 (2004)

29. Miwa, H., Fukuhara, T., Nishimura, T.: Service process visualization in nursing-care service using state transition model. Advances in the Human Side of Service Engineering, 312 (2012)

30. Nardi, B.A., Engeström, Y.: A web on the wind: The structure of invisible work. Comput. Support. Coop. Work. 8(1) 1--8 (1999)

31. Newman, M.W., Sedivy, J.Z., Neuwirth, C.M., Edwards, W.K., Hong, J.I., Izadi, S., Marcelo, K., Smith, T.F.: Designing for serendipity: supporting end-user configuration of ubiquitous computing environments. In $4^{\text {th }} \mathrm{ACM}$ conference on Designing interactive systems: processes, practices, methods, and techniques, pp. 147--156 (2002),

32. Nieminen, J., Mattelmäki, T.: Navigating in the World of Services-Visualizing a system of systems. Nordes, 4 (2011)

33. Orlikowski, W.J.: Knowing in practice: Enacting a collective capability in distributed organizing. Organ. Sci. 13(3) 249--273 (2002)

34. Paradi, J., Ghazarian-Rock, A.: A framework to evaluate video banking kiosks. Omega, 26 (4) 523--539 (1998)

35. Patrício, L., Fisk, R.P., Constantine, L.: Multilevel service design: from customer value constellation to service experience blueprinting. J. Serv. Res. 14(2) 180--200 (2011)

36. Reinders, M.J., Dabholkar, P.A., Frambach, R.T.: Consequences of forcing consumers to use technology-based self-service. J. Serv. Res. 11(2) 107--123 (2008)

37. Scott, M., DeLone, W.H., Golden, W.: IT quality and egovernment net benefits: a citizen perspective. In: ECIS 2011 Proceedings (2011)

38. Simonsen, J., Svabo, C., Strandvad, S.M., Hansen, O.E., Samson, K., Hertzum, M.: Situated design methods. Mit Press (2014) 
39. Sousa, R., Voss, C.A.: Service quality in multichannel services employing virtual channels. J. Serv. Res. 8(4) 356--371 (2006)

40. Star, S.L.: The Structure of Ill Structured Solutions: Boundary Objects and Heterogeneous Distributed Problem Solving. Distr. Artif. Int. 2, 37--54 (1989)

41. Suchman, L.: Making work visible. Commun. ACM, 38(9) 56--64 (1995)

42. Suchman, L.: Practice-based design of information systems: Notes from the hyperdeveloped world. Inform. Soc. 18(2) 139--144 (2002)

43. Suchman, L.: Human-machine reconfigurations: Plans and situated actions. Cambridge University Press (2007)

44. Surprenant, C.F., Solomon, M.R.: Predictability and personalization in the service encounter. J. Mark. 86--96 (1987)

45. Syrjänen, A., Kinnula, M., Kuutti, K., Sihvola, V.: A Facilitated VMC-Based Remote Service Kiosk Interface for Information Systems. In: Proceedings of IADIS Interfaces and Human Computer Interaction Conference, pp. 157--164 (2013)

46. Tax, S.S., McCutcheon, D., Wilkinson, I.F.: The service delivery network (SDN) a customercentric perspective of the customer journey. J. Serv. Res. 16(4), 454--470 (2013)

47. Vargo, S.L., Lusch, R.F.: The four service marketing myths remnants of a goods-based, manufacturing model. J. Serv. Res. 6(4), 324--335 (2004)

48. Voss, C.A., Hsuan, J.: Service architecture and modularity. Deci. Sci. 40(3), 541--569 (2009)

49. Wreiner, T., Mårtensson, I., Arnell, O., Gonzalez, N., Holmlid, S., Segelström, F.: Exploring Service Blueprints for Multiple Actors: A Case Study of Car Parking Services. In $1^{\text {st }}$ Nordic Conference on Service Design and Service Innovation. Oslo, Norway (2009)

50. Zomerdijk, L.G., Voss, C.A.: Service design for experience-centric services. J. Serv. Res. 13(1), 67--82 (2010) 